\title{
IMPLEMENTAÇÃO DO CHECKLIST DE CIRURGIA SEGURA EM UM HOSPITALUNIVERSITÁRTO
}

Adriana Cristina de Oliveira ${ }^{1}$, Arthur Rocha de Abreu², Stayse Soares de Almeida²

Objetivo: verificar a implementação do checklist de cirurgia segura entre equipes multiprofissionais pela auditoria de qualidade em um hospital universitário. Métodos: Estudo transversal e descritivo, realizado entre maio e julho de 2015, no centro cirúrgico de um hospital público e universitário. Foram acompanhadas cirurgias aleatórias, onde a dinâmica de aplicação do checklist pela equipe multiprofissional foi observada, comparando preenchimento do checklist conduzido com o preenchido pela equipe do estudo. Os dados foram analisados mediante distribuição, frequência relativa e percentual. Resultados: Na adesão do checklist, em $57 \%$ das cirurgias acompanhadas a identificação dos pacientes não foi realizada, $100 \%$ das equipes não se apresentaram no time out e não confirmaram o procedimento no Sign out. Conclusão: A proposta do checklist, de despertar a equipe para as etapas simples do procedimento cirúrgico, mas que, sem as quais, o potencial para eventos adversos (EA) é muito maior, não tem sido alcançada na prática clínica. Outro aspecto fundamental se refere à melhoria da comunicação entre a equipe multiprofissional que não tem sido contemplada no processo.

Descritores: Cirurgia, Lista de verificação, Segurança do Paciente

\section{IMPLEMENTATION OF SAFE SURGERY CHECKLIST IN A UNIVERSITY HOSPITAL}

Objective: verify the implementation of safe surgery checklist in a multidisciplinary team through process of quality audit in a university hospital. Methods: Transversal and descriptive study, carried out between May and July 2015, in the surgical center of a public and university hospital. The surgeries were randomly monitored, and the dynamics of checklist filling by a multidisciplinary team was observed, comparing checklist filling performed in operating room with the one filled out by the study team. The data were analyzed by distribution, relative frequency and percentage. Results: Regarding checklist compliance, in $57 \%$ of accompanied surgeries patients identification was not performed, $100 \%$ of the staff did not introduced themselves in "time out" and not even confirmed orally the procedure in "sign out". Conclusion: The checklist proposal of arousing staff for simple surgical procedure steps, but without which the potential for adverse events (AEs) is much larger, has not been achieved in clinical practice. Another important aspect is related to improvement of communication between multidisciplinary team which has not been contemplated in process.

Descriptors: Surgery, Checklist, Patient Safety

\section{PERCEPCIÓN DEL HOMBRE MAYOR EN RELACIÓN CON SU SEXUALIDAD}

Objetivo: Verificar la aplicación de la lista de verificación de cirugía segura entre los equipos multidisciplinarios para la calidad de la auditoría en hospital universitario. Métodos: Estudio transversal y descriptivo, realizado entre mayo y julio de 2015 , en el centro quirúrgico de un hospital público y universitario. Cirugías aleatorios se acompañan, donde se observó aplicación dinámica de la lista de verificación por parte del equipo. Los datos fueron analizados mediante distribución, frecuencia relativa y porcentual. Resultados: La adhesión de la lista de verificación, en 57\% de las cirugías acompañado la identificación de los pacientes no se realizó, $100 \%$ de los equipos no se presentó em tiempo y no confirmó el procedimiento cabo em signo. Conclusión: La lista de verificación propuesta, para despertar al equipo a los sencillos pasos del procedimiento quirúrgico, pero sin la cual, potencial de eventos adversos (EA) es mucho mayor, no se ha logrado en práctica clínica. Otro aspecto importante se refiere a mejora de la comunicación entre equipo multidisciplinario que no ha sido contemplado en proceso.

Descriptores: Cirurgía, Lista de Verificacion, Seguridad del Paciente 
O princípio "primum non nocere" (em primeiro lugar não causar danos), atribuido a Hipócrates há mais de 2000 anos, demonstra a preocupação desde a antiguidade com o risco envolvido na assistência à saúde, atualmente estabelecidos como eventos adversos associados à assistêncial. Os Eventos adversos (EAs) são definidos como lesão ou dano não intencional que podem resultar em incapacidade ou disfunção, temporária ou permanente, prolongando a internação ou morte como consequência do cuidado, e não pela evolução natural da doença de base ${ }^{2}$.

Frente ao exposto, em 2004 inicia-se nos países membros da Organização Mundial de Saúde (OMS), a Aliança Mundial para segurança do paciente, visando desenvolver ações conjuntas sob forma de campanhas denominadas Desafios Globais para a Segurança do Paciente, um deles denominado Cirurgias Seguras Salvam Vidas, objetivando elevar os padrões de qualidade de serviços de assistência à saúde $^{3}$. Estima-se que 234 milhões de procedimentos cirúrgicos são realizados no mundo a cada ano. Cerca de um milhão de pacientes morrem e sete milhões de pacientes desenvolvem complicações, dos quais $50 \%$ são considerados evitáveis. Um em cada 150 pacientes morre em consequência dos EAs, quase dois terços relacionados com a assistência médicohospitalar ${ }^{4}$.

Estudos apontam uma incidência de 38,4\% EAs em hospitais brasileiros,

e uma análise realizada em três hospitais de diferentes regiões mostrou que oito em cada 100 pacientes sofrem um ou mais EAs no ambiente cirúrgico. Dos EAs decorrentes da assistência, $67 \%$ são considerados evitáveis por meio de ações gerenciais ${ }^{5,6}$. Dentre estes considerados evitáveis, alguns deles são compreendidos como eventos inaceitáveis como: troca de paciente, cirurgia ou da lateralidade do procedimento a ser realizado.

Com o intuito de mitigar EAs, um checklist cirúrgico foi proposto pela OMS, visando auxiliar equipes na atenção a detalhes do procedimento cirúrgico, mas que, sem sua realização, podem ocasionar complicações durantes os procedimentos. O checklist consiste em três etapas para conferência de itens relacionados à segurança do procedimento: antes da indução anestésica (Sign in), antes da incisão cirúrgica (Time out) e antes de o paciente sair da sala de operações (Sign out).

No Sign Iin é verificada a identidade do paciente, marcação do sítio cirúrgico, assinatura do termo de consentimento e a conformidade dos materiais solicitados. Também são antecipadas as dificuldades de intubação e risco hemorrágico. No Time Out, todos os membros da equipe cirúrgica cirurgiões, anestesistas, enfermeiros - apresentam-se, antecipam possiveis complicações, confirmam verbalmente a identificação do paciente, sítio cirúrgico, e procedimento a ser realizado. Nessa etapa também são confirmados: (1) aplicação de antimicrobianos e tromboembólicos profiláticos: (2) conformidade dos exames de imagem, (3) funcionamento e a correta esterilização dos materiais. O Time Out é uma estratégia de assegurar a comunicação entre os membros da equipe e evitar erros como "paciente errado" ou "local errado". No Sign Out. o procedimento realizado deve ser novamente verbalizado, materiais usados conferidos e contados, amostras encaminhadas e planos pósoperatórios, discutidos. Nas três fases o coordenador do checklist deve confirmar se a equipe cirúrgica completou as tarefas para aquela etapa, antes de prosseguir para a nova fase $^{3}$.

No Brasil, estudos mostraram que os maiores problemas relacionados à adesão ao checklist estavam relacionados às etapas antes da indução anestésica e antes da incisão cirúrgica, exatos momentos onde a eficácia da implementação do checklist pode ser comprovada ${ }^{7.8}$.

Diante da importância desse tema, o presente estudo tem como pergunta norteadora a seguinte questão: A implementação do checklist de fato ocorre na prática conforme as orientações do protocolo para todas as suas etapas?

Assim, este estudo objetivou analisar, por meio de uma auditoria de processo, como a implementação do checklist cirúrgico ocorre na prática da equipe multiprofissional no centro cirúrgico de um hospital universitário de grande porte em Belo Horizonte. 


\section{METODOLOGIA}

Tratou-se de um estudo transversal e descritivo, conduzido no período de maio a julho de 2015, no centro cirúrgico de um hospital público e universitário, de 450 leitos, após sua aprovação pelo Comitê de ética em Pesquisa da Universidade Federal de Minas Gerais - COEP (CAAE:30782614.3.0000.5149)

O centro cirúrgico possui 13 salas de cirurgia, as cirurgias acompanhadas no presente estudo foram selecionadas de acordo com agendamento prévio verificado no mapa cirúrgico, independente do período: diurno, vespertino e noturno. Para coleta dos dados, uma cópia do checklist foi validada pela instituição, contemplando as três diferentes etapas - antes da indução anestésica (Sign in), antes da incisão cirúrgica (Time out) e antes do paciente sair da sala de operações (Sign out) - a fim de avaliar se estavam sendo seguidas ou não pelas equipes cirúrgicas. Para essa auditoria, um bolsista de iniciação cientifica foi apresentado como graduando que acompanharia o procedimento cirúrgico a fim de identificar suas etapas. A coordenação médica e de enfermagem do centro cirúrgico estavam cientes dessa auditoria. Assim, o aluno assistia a cirurgia do começo ao fim, com a finalidade de identificar como cada etapa do checklist estava sendo conduzida pela equipe cirúrgica. Após essa etapa de coleta, os dados foram lançados em planilha excel, analisados mediante distribuição, frequência relativa e percentual, os resultados apresentados em tabelas e gráficos.

\section{RESULTADOS}

No período de seguimento, 30 cirurgias foram acompanhadas, conforme distribuição no gráfico 1 .

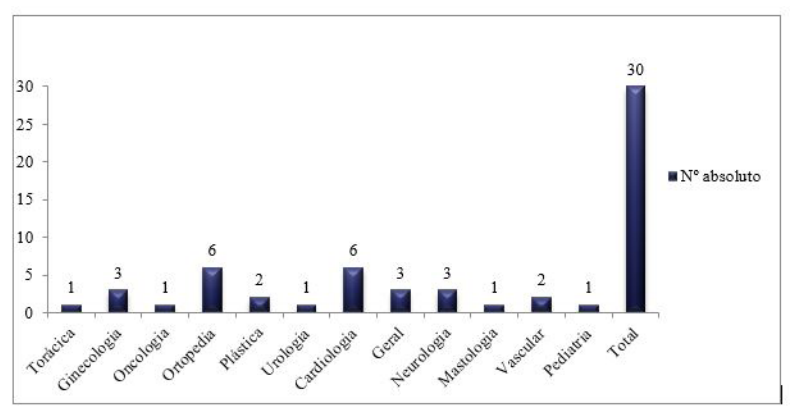

Gráfico 1 - Especialidades médicas cirúrgicas observadas em Hospital Universitário $(n=30)$. Belo Horizonte, 2016

Durante o seguimento dos procedimentos cirúrgicos como padronizado para o checklist os dados estão apresentados por etapas de aplicação. Assim a Tabela 1 apresenta variáveis relativas ao sign in - entrada do paciente no centro cirúrgico - referindo-se aos itens de verificação, identificação do paciente e procedimento a serem realizados antes do paciente ser anestesiado.
Destaca-se o alto percentual de não conferência na identificação do paciente, local e/ou do procedimento em $57 \%$ dos casos.

Tabela 1 - Auditoria do checklist para o sign in e avaliação da conformidade entre o protocolo adotado e o que de fato é conduzido pela equipe multiprofissional no centro cirúrgico $(n=30)$. Belo Horizonte, 2016.

\begin{tabular}{|c|c|c|}
\hline \multirow{3}{*}{ Itens avaliados } & \multicolumn{2}{|c|}{$\begin{array}{l}\text { Relação de conformidade entre o protocolo } \\
\text { adotado e o conduzido }\end{array}$} \\
\hline & Sim & Não \\
\hline & № absoluto (\%) & № absoluto (\%) \\
\hline $\begin{array}{l}\text { Termo de consentimento } \\
\text { assinado }\end{array}$ & 28 (93) & $2(7)$ \\
\hline $\begin{array}{l}\text { Identificação do paciente } \\
\text { e confirmação do } \\
\text { procedimento }\end{array}$ & $13(43)$ & $17(57)$ \\
\hline $\begin{array}{l}\text { Verbalização } \\
\text { (identificação do paciente, } \\
\text { local e procedimento) }\end{array}$ & $2(7)$ & $28(93)$ \\
\hline $\begin{array}{l}\text { Exames disponiveis para } \\
\text { consulta na Sala de } \\
\text { cirurgia }\end{array}$ & $29(97)$ & $1(3)$ \\
\hline $\begin{array}{l}\text { Materiais esterilizados } \\
\text { verificados por } \\
\text { indicadores }\end{array}$ & $24(80)$ & $6(20)$ \\
\hline $\begin{array}{l}\text { Antimicrobiano } \\
\text { administrado entre } 30 \text { e } \\
60 \text { minutos }\end{array}$ & $20(67)$ & $10(33)$ \\
\hline
\end{tabular}

Para a etapa do time out, em nenhuma das cirurgias constatou-se apresentação da equipe cirúrgica antes do procedimento cirúrgico. Outro ponto refere-se a estimativa das etapas críticas da cirurgia, explicitadas por 8 (27\%) das equipes nos procedimentos acompanhados.

Tabela 2 - Auditoria do checklist para o time out e avaliação da conformidade entre o protocolo e o que de fato é conduzido pela equipe multiprofissional no centro cirúrgico $(n=30)$. Belo Horizonte, 2016.

\begin{tabular}{|c|c|c|}
\hline \multirow{3}{*}{ Itens avaliados } & \multicolumn{2}{|c|}{$\begin{array}{l}\text { Relação de conformidade entre o protocolo } \\
\text { adotado e o conduzido }\end{array}$} \\
\hline & Sim & Não \\
\hline & № absoluto (\%) & № absoluto (\%) \\
\hline $\begin{array}{l}\text { Apresentação da equipe } \\
\text { cirúrgica }\end{array}$ & $0(0)$ & $30(100)$ \\
\hline $\begin{array}{l}\text { Monitorização da } \\
\text { temperatura do paciente }\end{array}$ & $3(10)$ & $27(90)$ \\
\hline $\begin{array}{l}\text { Controle de temperatura } \\
\text { dentro da sala de cirurgia }\end{array}$ & $23(76)$ & $7(23)$ \\
\hline $\begin{array}{l}\text { Etapas críticas da cirurgia } \\
\text { estimadas }\end{array}$ & $8(27)$ & $22(73)$ \\
\hline
\end{tabular}


No sign in, as amostras para anatomia patológica, do total de 12 cirurgias em que foram necessários sua realização, 10 (83\%) apresentavam identificação, enquanto 2 (17\%) das amostras não foram identificadas antes do paciente deixar a sala de operação. Relacionado ao controle da glicemia, quando o paciente é submetido a cirurgias cardíacas, das oito cirurgias observadas, todas elas relacionadas a troca de marca-passo, procedimento com uma média de duração de três horas, foram realizados o controle de glicemia em 2 (25\%) das cirurgias.

O gráfico 2 apresenta a ocorrência de falhas no preenchimento do checklist nas três etapas. O checklist foi integralmente conduzido pelo técnico de enfermagem, sem a

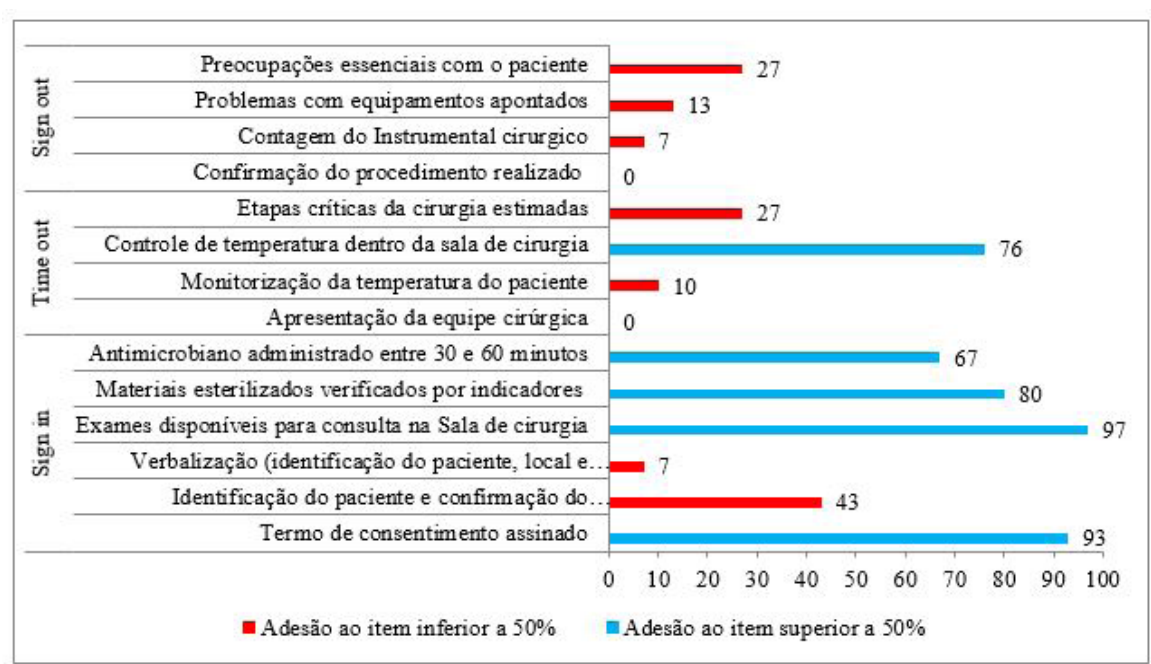

Gráfico 2 - Distribuição da adesão na aplicação do checklist cirúrgico em suas três etapas (sign in, time out e sign out) n= 30. Belo Horizonte, 2016. não tem sido alcançado na prática clínica.

Verificou-se ainda que a aplicação do checklist tem sido conduzida pela equipe de enfermagem, sem participação efetiva dos demais membros. Um dos pilares fundamentais da proposta do checklist para a mitigação dos EAs se refere especificamente a melhoria da comunicação entre a equipe multiprofissional, o que não foi observado durante a auditoria conduzida.

As dificuldades na segurança cirúrgica são reconhecidas em países desenvolvidos e em desenvolvimento, nestes últimos, sendo atribuidas à deficiência de infraestrutura, falhas na administração das organizações, às capacitações e treinamentos incompativeis com a realidade e ao subfinanciamento, predominando, no entanto, na maioria dos países a falha na comunicação entre a equipe multiprofissional'. Destarte, estudos apontam que quando há participação de todos os membros da equipe cirúrgica, melhorias na comunicação após aplicação do checklist são facilmente percebidas, evidenciando uma boa interação profissional e a definição clara das atividades de cada um $^{10,11}$

Outro EA considerado evitável refere-se a realização da cirurgia em local errado e no paciente errado?. No entanto, o que se verificou foi que a identificação de paciente e local não ocorreram na maioria dos procedimentos. Nos EUA, estimou-se que cirurgias em local errado e no paciente participação efetiva da equipe multiprofissional.

\section{DISCUSSÃO}

No seguimento dos 30 procedimentos cirúrgicos, sob perspectiva de avaliar como o checklist tem sido aplicado nas salas de cirurgia, verificouse que a aplicação do mesmo em suas três etapas, não tem atendido à sua premissa fundamental de melhoria da comunicação e participação efetiva de todos os membros da equipe multiprofissional. Por conseguinte, o despertar da equipe para as etapas simples do procedimento cirúrgico - mas que, sem as quais o potencial para EAs é maior errado ocorreram em cerca de 1 em 50.000 procedimentos, equivalente a 1.500 - 2.500 incidentes por ano. Em 2005, uma análise de 126 casos de cirurgias em sítio errado ou paciente errado revelou que $76 \%$ foram realizados no local errado, $13 \%$ no paciente errado ${ }^{12,14}$. Em 2009, foram publicados os resultados da implementação do checklist do Programa 'Cirurgias Seguras Salvam Vidas', no New England Journal of Medicine, revelando que as mortes provocadas por complicações cirúrgicas em local errado e no paciente errado foram reduzidas em quase a metade (de 1,5\% a $0,8 \%$ ). As boas práticas operações podem promover o empregadas na sala de 
sucesso da cirurgia, incluindo as checagens e demarcações para evitar procedimentos no paciente errado e local errado, evitando infecções no pós-operatório, diminuindo o tempo de internação e os custos hospitalares ${ }^{15,16}$.

Dentre os itens presentes no checklist e verificados na auditoria, a comunicação adequada entre a equipe multiprofissional durante o time out não foi realizada em nenhuma das cirurgias acompanhadas, com isso impedindose a possibilidade de correção de riscos associados aos EAs. Em um estudo que analisou 460 casos cirúrgicos com algum dano ao paciente durante o time out, em $22 \%$ desses a falha na comunicação foi o fator preponderante. Deste modo, ressalta-se a importância da comunicação no time out, de acordo com o preconizado pela OMS ${ }^{17-19}$

Estudos apontam ainda que o uso do checklist de segurança cirúrgica no time out reduziu infecções de sítio cirúrgico de $6,2 \%$ para $3,4 \%$, retorno não planejado para a sala operatória, de 2,4\% para $1,8 \%$ e $1,1 \%$ nas reoperações não planejadas ${ }^{19}$. Os achados do presente estudo, corroborados pela literatura, fortalecem a premissa que a implementação do checklist deve ser acompanhada periodicamente por auditoria de processo, em que se possam verificar potenciais aprimoramentos, evitando a falsa associação de que o preenchimento em sua totalidade está associado a redução das complicações.

Apesar da possibilidade dos seguimentos cirúrgicos analisados serem considerados quantitativamente reduzidos, não o consideramos uma limitação do estudo por se tratar de uma auditoria interna, o que na verdade revelou importantes aspectos a serem repensados na aplicação do checklist, assim como definir estratégias que favorecem uma maior participação da equipe multiprofissional e, como se ater com mais precisão ao fato de que contabilizar o preenchimento do checklist em uma instituição pode não traduzir exatamente o que ocorre durante o processo de sua aplicação, revelando uma realidade de confiabilidade questionável. O presente estudo pode contribuir ainda para delinear a realidade da adoção do programa Cirurgias Seguras Salvam Vidas nas instituições e para tal, somos favoráveis à sua expansão como projeto de vigilância seja em nível estadual e municipal em parcerias com as secretarias de saúde.

\section{CONCLUSÃO}

Verificou-se claramente pela auditoria que a implementação do checklist cirúrgico não tem ocorrido na prática da equipe multiprofissional conforme estabelecido pela coordenação do processo na instituição. Sua aplicação não tem atendido ao princípio fundamental de melhoria na comunicação entre os profissionais. As etapas, quando realizadas, não atendemao que de fato está proposto para cada uma delas, o que continua a corroborar para a insegurança do paciente. Foram despertados aspectos não considerados pelos profissionais, como a confirmação do procedimento a ser realizado no sign in, ou a estimativa das etapas críticas da cirurgia no time out, bem como a confirmação do procedimento no sign out.

Diante destes fatos, constata-se que a cultura de segurança do paciente visando a melhoria do cuidado ao paciente cirúrgico, ainda necessita de maiores investimentos na instituição, visando cumprimento das metas e os desafios globais propostos pela Organização Mundial da Saúde. Considerando a implementação do uso do checklist, independente da totalidade de seu preenchimento, constatou-se que sua aplicação não tem se traduzido na melhoria da comunicação ou a participação dos profissionais das diferentes categorias, configurando em uma necessidade básica da adoção do checklist que é a proposta de melhoria da comunicação.

Apoio: Fundação de Amparo a Pesquisa de Minas Gerais (FAPEMIG)

* Os autores agradecem ao bolsista de iniciação cientifica Everton Rocha Pacheco e da Enfermeira Miriam Cristina Assis Venâncio pela colaboração na etapa de coleta de dados. 


\section{REFERÊNCIAS}

1. Hanchanale V, Rao AR, Motiwala H, Karim OMA. Wrong site surgery! How can we stop it? Urology Annals. [Internet]. 2014 [cited 2016 Sep 13]; 6(1): 57-62. Available from: http://www.urologyannals.com/temp/ UrolAnn6157-3065453_083054.pdf

2. Jager E, Mckenna C, Bartlett L, Gunnarsson R, Ho YH. Postoperative adverse events inconsistently improved by the world health organization surgical safety checklist: a systematic literature review of 25 studies. World J of Surg. [Internet]. 2016 [cited 2016 Sep 13]. Available from: https://www.ncbi.nlm.nih.gov/pmc/articles/ PMC4943979/

3. Filho GRM, Silva LFN, Ferracini AM, Bähr GL. Protocolo de cirurgia segura da OMS: o grau de conhecimento dos ortopedistas brasileiros. Rev bras ortop. [Internet]. 2013 [cited 2016 Sep 13]; 48(6): 554-62. Available from: https://ac.els-cdn.com/ S2255497113001286/1-s2.0-S2255497113001286-main.pdf?_ tid=a033db6c-f6d6-1le7-b8de-00000aab0foleacdnat=1515678913_ d97fc4dfa0951b96c5aa88387bb5cd4d

4. Berrisford RG, Wilson IH, Davidge M, Sanders D. Surgical time out checklist with debriefing and multidisciplinary feedback improves venous thromboembolism prophylaxis in thoracic surgery: a prospective audit. Eur J Cardiothorac Surg. [Internet] 2012. [cited 2016 Sep 16]; 41(6): 1326-1329. Available from: https://academic.oup. com/ejcts/article/41/6/1326/416664

5. Souza LP, Bezerra ALQ, Silva AEBC, Carneiro FS, Paranaguá TTB, Lemos LF. Eventos adversos: instrumento de avaliação do desempenho em centro cirúrgico de um hospital universitário. Revista enfermagem UERJ [Internet]. 2011 [cited 2016 Sep 21]:19(1):127-13. Available from: http://www.facenf.uerj.br/v19nl/v19nla2l.pdf.

6. Gimenes FRE, Marques TC, Teixeira TCA, Mota MLS, Silva AEBC, Cassiani SHB. Medication wrong-route administrations in relation to medical prescriptions. Rev. Latino-Am. Enfermagem. [Internet]. 2011 [cited 2016 Sep 21]; 19(1): 11-7. Available from: http://www.scielo.br/pdf/ rlae/v19nl/pt_03.pdf

7. Muniz RV, Bohrer BBA, Mulazzani MP, Erig LS, Santos SM, Santos HB. Criação e evolução da lista de verificação de cirurgia segura especifica para oftalmologia. Revista Acreditação: ACRED. [Internet]. 2015 [cited 2016 Sep 13]; 5(9). Available from: http://dialnet.unirioja.es/servlet/ articulo?codigo $=5626618$.

8. Martins GS, Carvalho R. Realização do time out pela equipe cirúrgica: facilidades e dificuldades. Revista SOBECC. [Internet]. 2014 [cited 2016 Sep 16]; 19(1). Available from: https://revista.sobecc.org.br/ sobecc/article/view/49.

9. WHO. World Health Organization. World Health Organization: World Alliance for Patient Safety. Taxonomy. The Conceptual Framework for the International Classification for Patient Safety. Final Technical Report. January 2009, version 1,1. [cited 2016 Ago 12]. Available from: http://www.who.int/patientsafety/taxonomy/icps_full_report.pdf.
10. Cabral RA, Eggenberger T, Keller K, Gallison BS, Newman D. Use of a Surgical Safety Checklist to Improve Team Communication. AORN Journal. [Internet]. 2016 [cited 2016 Sep 16]: 104(3): 206-16. Available from: file:///C:/Users/Usuario/Downloads/Cabral_et_al-2016AORN_Journal.pdf

11. Pancieri AP, Santos BP, Avila MAG, Braga EM. Checklist de cirurgia segura: análise da segurança e comunicação das equipes de um hospital escola. Rev Gaúcha Enferm. [Internet]. 2013 [cited 2016 Sep 16]; 34(1):71-8. Available from: http://www.scielo.br/pdf/rgenf/ v34nl/09.pdf.

12. Treadwell JR, Lucas S, Tsou AY. Surgical checklists: a systematic review of impacts and implementation. BMJ Qual Saf. [Internet]. 2013 [cited 2016 Sep 16]. Available from: http://qualitysafety.bmj.com/ content/qhc/23/4/299.full.pdf

13. Mehtsun WT, Ibrahim AM, West MD, Pronovost PJ, Makary MA. Surgical never events in the United States. Surgery. [Internet]. 2013 [cited 2016 Sep 16]; 153(4): 465-72. Available from: http://doi. org/10.1016/j.surg.2012.10.005.

14. Santana HT, Siqueira HN, Costa MMM, Oliveira DCAN, Gomes SM, Sousa FC, et al. A segurança do paciente cirúrgico na perspectiva da vigilância sanitária - uma reflexão teórica. VISA em Debate: Sociedade, Ciência \& Tecnologia. [Internet]. 2014 [cited 2016 Sep 25]; 2 (2). Available from: http://periodicos.fiocruz.br/pt-br/publicacao/8351.

15. Haynes AB, Weiser TG, Berry WR, Lipsitz SR, Breizat AHS, Dellinger EP, et al. A surgical safety checklist to reduce morbidity and mortality in a global population. N Engl J Med. [Internet]. 2009 [cited 2016 Sep 25]: 360(5): 491-99. Available from: http://doi.org/10.1056/ NEJMsa0810119.

16. Fourcade A, Blache JL, Grenier C, Bourgain JL, Minvielle E. Barriers to staff adoption of a surgical safety checklist. BMJ Quality \& Safety. [Internet]. 2011 [cited 2016 Sep 23]: 21(3): 191-7. Available from: http:// doi.org/10.1136/bmjqs-2011-000094.

17. Griffen FD. ACS Closed Claims Study reveals critical failures to communicate. Bull Am Coll Surg. [Internet]. 2007 [cited 2016 Sep 28]; 92(1):11-6. Available from: https://www.ncbi.nlm.nih.gov/ pubmed/17375480.

18. Maziero ECS. Avaliação da implantação do Programa Cirurgia Segura em um hospital de ensino [dissertação] - Curitiba (PR). Universidade Federal do Paraná; 2012 [cited 2016 Sep 25]. Available from: http://acervodigital.ufpr.br/handle/1884/29376.

19. Guzzo GM, Guimarães SM, Magalhães AMM. Efeitos e desafios da implantação de um sistema de verificação de segurança cirúrgica: revisão integrativa. J Nurs Health. [Internet]. 2014 [cited 2016 Sep 28]; 4 (2):155-64. Available from: https://periodicos.ufpel.edu.br/ojs2/ index.php/enfermagem/article/view/3393. 